\title{
اثر اسعار النفط على مؤشرات الاسهم الاساسية، دراسة في عينة من الدول المثقدمة للمدة2007-2020
}

\author{
م.ح. دلمان كامل احمد، قسم الاقتصاد، جامعة نوروز، أقليم كوردستان العراق
}

يدور البحث حول الاهمية التي تلعهاكل من النفط باعتباره المصدر الرئيسي_ للطاقة ومؤشرات الاسواق الماليـة العالمية كونها يؤثران ويتأثران بالاقتصاد في ظل ترابط وتشـابك جميع القطاعات

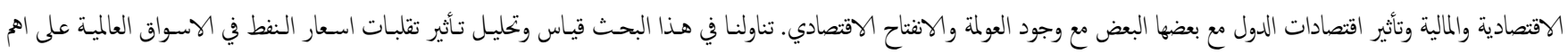

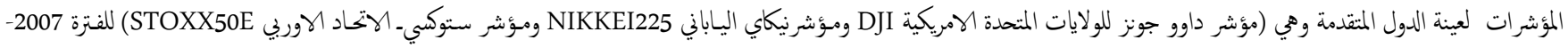

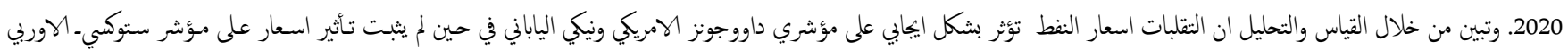

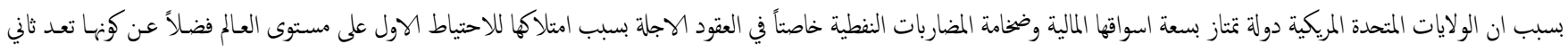
اكبر مستورد.

الكلمات المفتاحية: اسعار النفط، مؤشرات الاسهم، مؤشر داوو جونز ، مؤشر نيكي، مؤشر ستوكي.

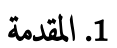

النتيض من ذلك عادةً ماتنشأ الانار قصير الاجل على الاسوق المالية. ويؤثز التقلبات في اسعار النفط تأثيراً كيبراً على اسعار الاسهم والارباح في الاقتصادات المتقدمة والنامية. وفقاً لحجة بارك وراتي (2008)، اذا كانت التغيرات المفاجئة والقاسية في اسعار النفط قادرة على التأثير على الاقتصاد الحقيقي بسبب سلوك المستهلك الراسخ. فأن هذه النتاجُ ستؤثر على سوق الاسهم العالمية.

1.1

ان العلاقات بين المتغيرات الاقتصادية المالية والحقيقية ذات تاثيرات متبادلة وغالبا ما تنتقل الاثار والتقلبات الناشئة عن الدورات الهقتصادية من القطاع الملالي الى القطاع الحقيقي والدلائل التاريخية المرتبطة بالازمات التي حدثت في القرنين الاخيريين تشير الى ان الازمات غالبا ما تبدء في القطاع المالي ومن ثم تنتقل الى القطاع الحقيقي ومع تطور الاسواق المالية وتول الكثير من السلع الى ادوات مالية مشتقة اصبح من الممكن ان يكون الناثير معاكسا كما هو الحال في النفط مثلا الذي يمارس تاثيرات محمة على القطاع المالي ويتاثر بيه.

2.1

يفترض البحث ان اسعار النفط كتغير مستقل تؤثر طرديا على مؤشرات الاسواق المالية في الدول المتقدمة (عينة الدراسة).

3.1

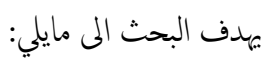
اولا: اعطاء اطار نظري عن اتجاهات النفط الدولية وكذلك عن المؤشرات يعتبر مادة النفط الخام واحدة من اهم المواد الرئيسة التي تدخل في الكثير من الصناعات. ولكن تظهر الهمية الكبيرة للنفط الخام من خلال كونها المصدر الرئسي لتوليد الطاقة، لنا نزى في الوقت الحاضر الاهمية الكبيرة للنفط على الاقتصاد العالمي، سواءكان لدول المنتجة والمصدرة او الدول المستهلكة لهذا المادة

$$
\text { الطبيعية المحدودة والناضبة في باطن الارض. }
$$

ومن الواضح أن للنفط له تأثير كير على الاقتصاد العالمي. ووفقا للدراسات الحديثة وهذا مأكدته منظمة الطاقة الدولية في تقريرها في عام 2020، فإن تأثير أسعار النفط على الاقتصاد هو أهم مصدر قلق للاقتصاديين و للسياسين و اصحاب القرار في الوقت الحاضر. ولا تفرق الدراسات السابقة بين البلدان المصدرة للنفط والبلدان المستوردة للنفط عندما قامت بالتحقيق في آثار تقلبات أسعار النفط على عائدات الاوراق المالية. من الممكن ان تؤثر اسعار النفط الخنام على الاقتصادات من خلال ثلاث قنوات رئيسة:- اولاً:- ان التغيرات في اسعار النفط الخام وخاصتاً نخو الارتفاع، لها تأثير قوي على التضخم من خلال التغبرات في تكاليف الانتاج بالنسبة للبلدان الصناعية، مما يؤدي الى تحول في منحنى العرض. ثانياً:- بالنسبة للبلدان وخاصتاً المستوردة للنفط، فأن النغيرات في اسعار النفط الخام وتحديداً التغير نحو الانخفاض تؤثر تأثيراً كبيراً على ميزان المدفوعات. وعلاوة على ذلك، فن المتوقع ان تؤدي اسعار النفط دوراً هاما في هذه العملية نظراً لان التضخم والتوازن التجاري ها عاملان هامان محددان لاسعار الصرف في الاجلين المتوسط والطويل. وعلى 


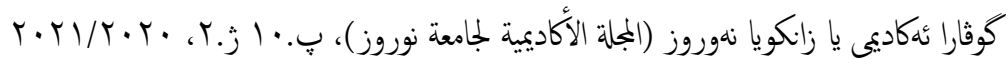

الدول المتقدمة والنامية مختارة على العملات ويظهر في الدراسة ان العملات تظهر تبعية كبيرة لاسعار خام. وان العلاقة تعزز بشكل ملحوظ بعد الازمة المالية 2008 والخغفاض اسعار النفط في عام 2014. وان اعلى مستوى من الاعتماد يكون على العملات البلدان المثقدة والمصدرة للنفط يليه تصدير البلدان النامية المصدرة للنفط في حين ان البلدان النامية المستوردة للنفط يوجد لديها اقل الاعتمادوقد تم اكتشاف ان مؤشرات سوق الاسهم في العديد من البلدان كان له تأثير كير (ايياني) على اسعار خام التكساس لهوولاء البلدان، وكان قيم الاعتماد على الطاقة بشكل كبير مقارنتا بالمصدرين. رسالة ماجستير اكلاديي (بالنور هاجر ، منصر سامية، فاطمة بوجريدة)، 2019-2018: بعنوان (لتحقق اثر تقلبات اسعار النفط على عوائد مؤشرات الاسواق المالية دراسة قياسية بين الدول المصدرة (المملكة العربية السعودية) والدول المستوردة (فرنسا) خلال الفتزة 2008-2018) قام بها ، توصل الباحثون الى ان السوق الفرنسي تحقق عوائد مالية اكبر

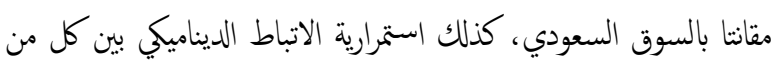
عوائد السوقين الفرنسي والسعودي وايضاً عوائد تقلبات اسعار النفط باستمرار لفترة طوياة.

Starvros Degiannakis, George filis, Renatas دراسة للباحثين The Effects of Oil ) بعنوان 2014, vol. Volume 35 ,Kizys Price Shocks on Stock Market Volatility: Evidence from (European Data النفطية اسهم اسواق المالية، فتوصلوا المى نتائج توضح وجود تاثير ايجابي لصدمات اسعار النفط بالنسبة لاسهم شركات الطاقة في السوق الملالي الاوريي. من الشكل اعلاه نلاحظ تقلبات كثيرة في اسعار النفط الخام خلال الفترة الزمنية لسلسلة البيانات التي اخذناها للبحث ، ومن اهها : ان اول ارتفاع لسعر النفط الخام لعام 2007 بدء في اكتوبر وديسمبر ليصل الى ( 94,53 - 95,48 دولار) على التوالي ويرجع سبب هذا الارتفاع الى زيادة الطلب العالمي على النفط في هذه الفترة بصورة كبيرة جدا بعد نمو الطلب في الهند والصين وباقي الدول النائة (بالنور هاجر، منصر سامية، فاطمة بوجريدة،2018-2019). وان الطلب كان مفاجئا ولم تكن منظمة اوبك جاهزة له
الاساسية اسواق امريكا واوربا واليابان باعتبارها بمعيا من الدول المتقدمة.

ثانيا: قياس وتحليل اثر اسعار النفط على بعض المؤشرات اسواق المالية

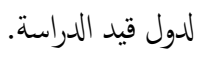

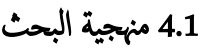

يتبع البحث المنهج الاستقرائي من خلال انتقال الى نتأُّكلية من مقدمات جزئية مع استخدام اساليب قياسية والبرنامج الاحصائي لتحديد تاثير المتغير المستقل على المتغيرا التابع للمدة 2007-2020.

\section{1}

يتالف البحث من جانبين ها الجانب النظري والذي ينقسم بدوره الى دراسات سابقة وتحليل اتجاهات اسعار النفط وشرح بشكل مختصر لبعض المؤشرات الاسواق المالية المختارة. والجانب الثاني يمثل بيان الثاثير بين اسعار النفط

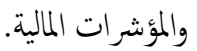

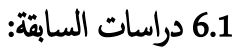

رسالة (Taheri,Noushin) في 2014: جائت رسالة الماجستير بعنوان The Impact of Oil Price on Stock Markets: Evidence ) من خلال البحث تم تأيكد تأثير اسعار (from Developed Markets النفط على الاسواق الاوراق المالية في كل من (المملكة المتحدة, كندا، فرنسا، والولايات المتحدة الامريكية) واستخدام الباحث متغيرات اخرى مثل الانتاج الصناعي ومعدل الفائدة الحقيقي في دراسته. بأستخدام البيانات الشهرية للفترة تتراوح بين عام 1990: 01 إلى 2012: 12. وكان الهدف الأول من الدراسة هو فهم سلوك البلدان المنتجة للنفط والبلدان المستهلكة للنفط. ووفقا للاختبارات، كانت استجابة السواق الووراق المالية لكندا كنتج للنفط لزيادة أسعار النفط إيهابية، وقد ظهر الأثر في الشهر الأول. أما البلدان الباقية التي كانت مستهلكة للنفط فتستجيب لهذا

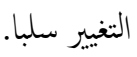

دراسة اخرى قام بها الباحثون ( Derya Ezgi Kayalar a, C. (Coşkun Küçüközmen b, A. Sevtap Selcuk-Kestel The impact of ) بعنوان :.January 2017, Pages 162-173 crude oil prices on financial market indicators: Copula (approach 


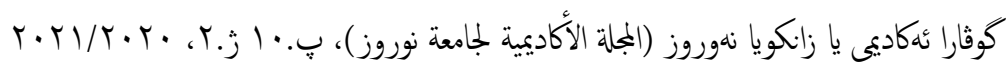

العالمية جراء الازمة المالية والاقتصادية التي بدءت في الولايات المتحدة الهمريكية

لتشمل بعدها الدول الاخرى والاقتصاد العالمي (الشطي،2014).

استمرت اسعار النفط الحام في تقلباتها مابين ادنى مستوى لها في عام 2009 بسعر ( 41.68 دولار) الى اعلى مستوى نهاية عام 2010 بسعر (113.92 دولار ) . و و بعد التعافي من الازمة العالمية وعودة اسعار النفط الى الارتفاع انخفضت في شهر مارس عام 2010 من (86.15 دولار) الى (73.97دولار) للبرميل بسبب قيام ناييريا بزيادة طاقتها الانتاجية بعقدار 4 ملايين برميل في اليوم الواحد (Odupitan, (2017) بشكل عام تباطئ نمو الناتج المحلي الإجلالي العالمي الحقيقي في عام 2010 و قد يكون تباطؤ النمو العالمي قد مارس ضغطًا هبوطيًا على أسعار بعض السلع الأنماط الحديثة مثل النفط (Ellwanger, Zmitrowcz, 2014) في اخر شهر من عام 2010 شهدت اسعار النفط صعود اخر بسبب زيادة الطلب من قبل دول شرق اسيا والصين والهند مع وجود الاضطرابات السياسية في دول شرق الوسط (سمير سعيفان، 2015). ان تفامٌ الاضطرابات السياسية في بعض الدول العربية عام 2011 بسبب ثورة الربيع العربي أدى ذلك إلى انقطاع الكثير من الامدادات النفطية لهذه الدول منها ليبيا وسوريا واليمن. وخرج نخو 1.6 مليون برميل يوميا من انتاج النفط اللبيي ولم تتمكن اعضاء اوبك الاخرى من تعويضه مما تسبب في ارتفاع أسعار النفط منذ ذلك الحين فوق مستوى ( 100 دولار) . وبسبب فرض حظر التصدير من قبل كل من الولايات المتحدة الامريكية والاتحاد الاوربي للنفط الايراني ادى المى خروج نخو مليون برميل من النفط الخام لها يوميا في الاسواق. وهذا الامر تسبب بدوره الى زيادة الخخاوف من رد فعل عسكري لدى الحكومة الايرانية في ذلك الوقت وهذا عامل اخر ابقى الاسعار النفطية مرتغعة بشكل كير قياسا بالارتفاعات (الحقباني، محمد، 2017). في عام 2012 سجل اعلى ارتفاع في اسعار النفط الخام في شهر فبراير بسعر (107 دولار) للبرميل الواحد وكان وراء هذا الارتفاع في اسعار النفط الخام العديد من الاسباب ، منها انخفاض انتاج ايران بحدود 700 الف برميل يوميا . وقد دفت العقوبات بعض من الشركات التأمين في الاتحاد الاوروبي من تقديم تغطيات تأمينية للصادرات وهذا بدوره اعاق واردات بعض المشترين من خارج دول الاتحاد الاوروبي ايضا ومنهم كوريا الجنوبية عادو لشراء الخام الايراني حيث وجدو صعوبة في الوفاء بمواعيد تسلمه بسبب صعوبات تعرض لها اسطول وهو السبب الرئسي في ارتفاع الاسعار بسبب محدودية نمو العرض النفطي امام نمو الطلب (فروج ، 2015). وكان هذا الارتفاع بشكل تذبذب في بعض الاشهر منذ بداية عام 2007 الى ان وصل الى اعلى سعر خلال عقود لنتجاوز 140 دولار في منتصف عام 2008 وكان هذا ثاني اكبر ارتفاع لاسعار النفط في تاريخه بعد الارتفاع الاول في بداية السبعينيات بسبب الصدمة النفية الاولى.

\section{Chart Title}

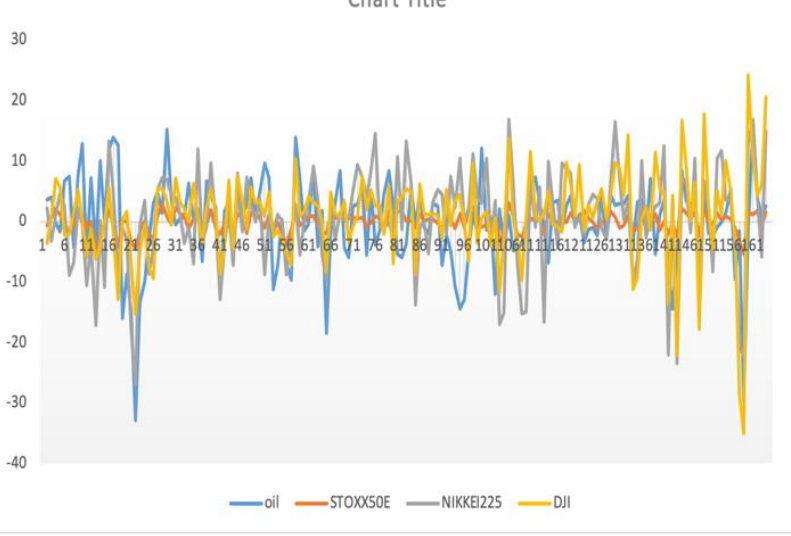

الرسم من عمل الباحث بالاعتمد على:- IMF, Data base, international financial statistic (IFS)

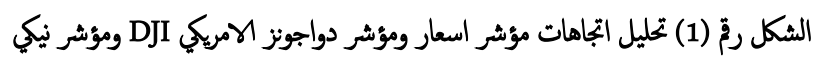

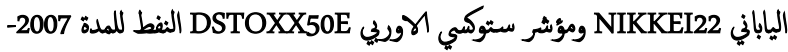
2020

لكن في شهر حزيران من نشس العام بدء مشوار الاخغفاض لاسعار النفط ولم يعد الى هذا الحد مرة خرى لحد الان. فني ذلك الشهر انخضض سعر البرميل من 140 دولار للبرميل الواحد ليصل الى (115,46- 124,08) دولار الشهري تموز وأب على التوالي لعام 2008 ويرجع ذلك لاسباب عديدة منها تحول تاثير الازمة المالية العالمية المى القطاعات الاقتصادية الاخرى اي القطاع الحتيقي، كذلك كانت من الأسباب المهمة لانهيار في أداء الأسواق المالية العالمية والمضاربات فها, ومن ثم تأثر الفرق بين العرض والطلب، وبجانها التأثيرات الجيوسياسية.( إبههي،

(2016

استمر الهبوط في اسعار النفط كما ذكرنا ليصل الى 41.68 دولار للبرميل في بداية عام 2009 والسبب هوالفرق بين نمو الطلب المتزايد لتزاجع الضغط على منظمة الدول المصدرة للنفط ( اوبك) لضخ المزيد. والسبب الاخير يرجع للمضاربات بالنفط في الاسواق العالمية (نوش،2008). ثم اخذت اسعار النفط بالانعاش مرة اخرى نخو الارتفاع ليكون اعلى سعر تصل اليه في اخر شهر من نفس السنة بحدود (80 دولار) مستغلة في ذلك ضعف الدولار الأمريكي وقوة أداء الشركات الأمريكية. كذلك القرار الذي اتخذه اعضاء اوبك بتخفيض انتاجما بمقدار 4.2 مليون برميل يوميا بسبب ضغوطات هبوط اسعار النفط في الاسواق 


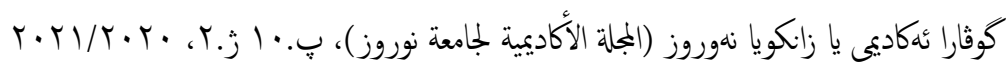

انخضض واستمر التذبذب لتسجل اعلى مستوى في الشهر الاخير لعام 2016 بسعر 55.72 دولار للبرميل الواحد (Bulletin, 2018) شهد الربع الاخير من عام 2018 انخفاضا في سعر النفط العالمي لكن كان الانغاض في الشهر الاخير من نفس العام اكثر حدة ويرجع سبب هذا الانخاض الى مخاوف من زيادة العرض العالمي بالتزامن مع النمو الاقتصادي المتزاجع (2019, PETROLEUM ECONOMIST). وفي الشهر الوول من عام 2019 ارتغ سعر برميل النفط بشكل مطرود ليتجاوز 60 دولار في شهري نيسان وايار وعاد المى الاخخفاض مرة اخرى لكن بشكل طفيف الى ان بلغ ادنى مستوياته نهاية عام 2019 وبداية عام 2020 ليواجه العالم جائحة كورنا والتي كانت سبب في حدوث ازمة اقتصادية عالمية وتبعاتها من ركود وبطالة وتوقق اغلب الانشطة الاقتصادية في عموم الدول (اوبك, 2020).

2. - 2 - الاختبارات القياسية

جدول 1: :نتاجُ اختبار ديكي فولر (ADF)جلذر الوحدة للمتغيرات المدروسة للمدة

(2020-2007)

\begin{tabular}{|c|c|c|c|c|}
\hline \multicolumn{2}{|c|}{ الفرق الأول } & \multicolumn{2}{|c|}{ المستوى } & \multirow{2}{*}{ المتغيرات } \\
\hline t الجدولية t t & t المتسبة & t المتسبة & t الجدولية t & \\
\hline \multirow[t]{2}{*}{-3.43780} & - & - & - & oil price سعر النفط \\
\hline & 9.96375 & 3.34489 & 3.437801 & \\
\hline \multirow{2}{*}{-2.8792} & -12.029 & - & -3.4706 & مؤشر داو جونز (DJI) \\
\hline & & 0.3244 & & \\
\hline-2.8792 & -11.858 & -0.6067 & -3.4704 & نيكاي 225 (N225) \\
\hline-3.4709 & -11.414 & -2.3857 & -3.4706 & 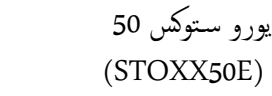 \\
\hline-3.4709 & -12.673 & -1.6083 & -3.4709 & $\begin{array}{r}\text { الناتج المحلي الاجلي الامريكي_GDP } \\
\text { USA }\end{array}$ \\
\hline-3.4709 & -6.5019 & -1.1575 & -3.4709 & $\begin{array}{r}\text { الناتج المحلي الاججالي الياباني } \\
\text { JAPAN_GDP }\end{array}$ \\
\hline-3.4709 & -3.8002 & -3.1087 & -3.4709 & الناجت المحلي الاجالي للاتحاد \\
\hline & & & & EUR_UNION_GDP \\
\hline
\end{tabular}

بالنظر المىنتأُج اختبار ADF لجذر الوحدة الموجودة في الجدول رڤٌ (1)، حيث تم تطبيق الاختبار للمتغيرات المشمولة بالدراسة وهي ( اسعار النفط، مؤشر داوجونز ، مؤشر نيكلي الياباني، مؤشر ستوكي الاوربي، والناتج المحلي الاجلالي لكل من الولايات المتحدة الامريكية والاتحاد الاوربي واليابان)، بينت نناجُ كرئ الاختبار بتطبيق ان جميع المتغيرات لم تكن ساكنة عند المستوى، وبتطبيق الاختبار على الفرق الاول للمتغيرات غير الساكنة عند المستوى العام كثفت
الناقلات الايرانية مما تسبب في ابطاء الصادرات. وكذلك نتيجة التطورات الجيوسياسية في المنطقة العربية (مراد، 2017). وفي شهر ايار من عام 2012 نلاحظ هبوط اسعار النفط مرة اخرى حيث انخضت من 104.87 دولار في شهر ابريل الى 86.96 دولار في شهر مايو اي انخفاض بحدود 19 دولار بسبب زيادة الصادرات النفطية للحكومة الجزائرية حيث ارادت ان تزيد من ايراداتها النفطية (مراد، 2017). والارتفاع الاخر كان في بدايه اغسطس سنة 2013 اغسطس حيث سجل اعلى ارتفاع لسعر النفط الخام بحدود ( \$75) مقارنة بالشهر الذي سبقه ويرجع سبب هذا الارتفاع المى بداية الحرب الاهلية في ليبا وفرض العقوبات على ايران بسبب الشكوك عليها بشأن نواياها النووية في اوائل عام 2012. وكذلك الانقطاعات الطفيفة في الانتاج في كل من السودان ونيجيريا وسوريا وبحر الشمال (2015 Jodiekeane, Willem,) استمر ارتفاع الاسعار النفطية في الاسواق العالمية بعد منتصف عام 2012 بشكل تدريي ليصل الى اعلى مستوى له في اغسطس لعام 2013 بسعر 107

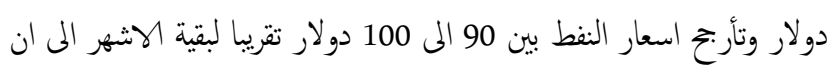
وصلنا الى شهر اكتوبر في عام 2014 لينخفض الى 11 دولار اي بسعر 80 دولار تقريبا وهذا الانخاض كان نقطة الانقلاب نحو الاخغفاض الحاد بالنسبة لاسعار النفط لنزى في الثهر الذي تلاه انخفاض اكبر ومن ثم انخفاض مستمر ليصل الى ادنى مستوى له في اول شهر من عام 2016 ، ويرجع سبب هذا الانخفاض الاسباب عديدة لكن من اهها قيام الولايات المتحدة الامريكية بزيادة انتاجما من النفط بمقدار 1.2 مليون برميل يوميا والتي تمثل الزيادة بمقادر 16.4\% وهي اكبر زيادة في الحجم منذ عام 1990 ، وكذلك يشير تحليل البنك الدولي الى زيادة صادرات النفط العراقي ثاني اكبر منتج في اوبك والتي بلغت اعلى مستوياتها منذ عام 1990 حيث بلغ متوسط 2.9 مليون برميل يوميا في الشهر الثالث لعام 2015 ـ واخير عامل الصدمات لهذا الانخفاض هو ارتفاع الدولار الامريكي الذي اصاب سوق النفط قبل يونيو 2014 (Bulletin, (2018 وبعد انخفاض اسعار النفط عالميا منذ اواخر عام 2014 ليصل الى ادنى مستوياته وهو 33.62 دولار للبرميل خلال العامين ( 2015 - 2016) حيث شهدت اول حركة ارتفاع طفيف لاسعار النفط منتصف عام 2016 وبعدها 


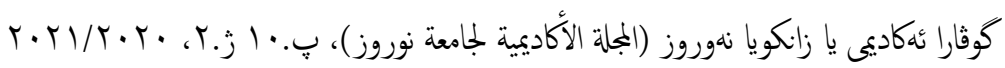

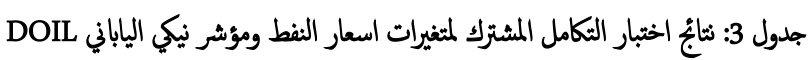
NNIKKEI22 الناجّ الملي الاجلي الياباني للمدة (2007-2020)

\begin{tabular}{|c|c|c|c|c|}
\hline \multicolumn{5}{|c|}{ Test (Trace) $\boldsymbol{\lambda}$} \\
\hline Eigenvalue & $\begin{array}{c}0.05 \\
\text { Critical } \\
\text { Value }\end{array}$ & $\begin{array}{l}\boldsymbol{\lambda}_{\text {trace }} \\
\text { Statistic }\end{array}$ & Prob. $^{* *}$ & $\begin{array}{c}\text { Null } \\
\text { hypothesis }\end{array}$ \\
\hline 0.125499 & 29.79707 & 31.58259 & 0.0308 & $\mathrm{r}=0$ \\
\hline 0.160068 & 15.49471 & 18.26031 & 0.0412 & $\mathrm{r}=0$ \\
\hline 0.032579 & 3.841466 & 0.410559 & 0.5217 & $\mathrm{r} \leq 1$ \\
\hline \multicolumn{5}{|c|}{ Maximum Eigenvalue $\boldsymbol{\lambda}$ Max } \\
\hline Eigenvalue & $\begin{array}{c}0.05 \\
\text { Critical } \\
\text { Value }\end{array}$ & $\begin{array}{l}\boldsymbol{\lambda} \text { trace } \\
\text { Statistic }\end{array}$ & Prob. $^{* *}$ & $\begin{array}{c}\text { Null } \\
\text { hypothesis }\end{array}$ \\
\hline 0.125499 & 21.13162 & 21.32228 & 0.0470 & $\mathrm{r}=0$ \\
\hline 0.160068 & 14.26460 & 19.84975 & 0.0220 & $r=0$ \\
\hline 0.032579 & 3.841466 & 0.410559 & 0.5217 & $\mathrm{r} \leq 1$ \\
\hline
\end{tabular}

وبالنظر المى جدول رقز (3) لنتائج اختبار جوهانسن للتكمل المشترك باستخدام دالة الامكان الاعظم لمتغيري اسعار النفط ومؤشر نيكي الياباني و_الناتج المحلي الاجلالي الياباني، بينت النتائُ ان القيمة المحسوبة لكل من اختبار احصاءة الاثر بلغت (trace=31.58259) وهي اكبر من القيمة الحرجة عند مستوى معنوية 5ax و وكما موضح في الجدول(3) اما فيا يخص اختبار قيمة العظمى eigenvalue فقد بلغت احصاء القيمة العظىى (21.32228 ) وهي اكبر من القيمة الحرجة عند مستوى معنوي 5\%، اي اننا في الاختباريين نرفض فرضية العدم تفتزض ان r=0، ونتبل بفرضية البديلة التي تنص على وجود تكامل مشترك.

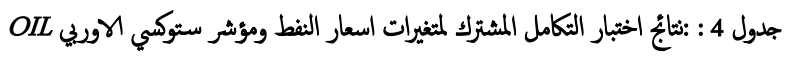
DSTOXX50E

\begin{tabular}{|c|c|c|c|c|}
\hline \multicolumn{5}{|c|}{ Test (Trace) $\boldsymbol{\lambda}$} \\
\hline Eigenvalue & $\begin{array}{c}0.05 \\
\text { Critical } \\
\text { Value }\end{array}$ & $\begin{array}{l}\boldsymbol{\lambda}_{\text {trace }} \\
\text { Statistic }\end{array}$ & Prob. $^{* *}$ & $\begin{array}{c}\text { Null } \\
\text { hypothesis }\end{array}$ \\
\hline 0.094694 & 29.79707 & 30.48350 & 0.0416 & $r=0$ \\
\hline 0.061032 & 14.49471 & 15.66591 & 0.0564 & $r=0$ \\
\hline 0.028841 & 3.841466 & 4.653093 & 0.0310 & $\mathrm{r} \leq 1$ \\
\hline \multicolumn{5}{|c|}{ Maximum Eigenvalue $\boldsymbol{\lambda}$ Max } \\
\hline Eigenvalue & $\begin{array}{c}0.05 \\
\text { Critical } \\
\text { Value }\end{array}$ & $\begin{array}{l}\boldsymbol{\lambda}_{\max } \\
\text { Statistic }\end{array}$ & Prob. $^{* *}$ & $\begin{array}{c}\text { Null } \\
\text { hypothesis }\end{array}$ \\
\hline 0.094694 & 19.13162 & 21.81760 & 0.0357 & $\mathrm{r}=0$ \\
\hline 0.061032 & 14.26460 & 10.01281 & 0.2111 & $\mathrm{r}=0$ \\
\hline 0.028841 & 3.841466 & 4.653093 & 0.0310 & $\mathrm{r} \leq 1$ \\
\hline
\end{tabular}

النتائُ عن عدم وجود جذر الوحدة، وعليه يكن الاستنتاج بان السلاسل الزمنية المستخدمة في الموذج هي سلاسل غير ساكنة عند المستوى ولكنها ساكنة عند فرقها الهول، وكل متغير على حده يعد متكملا من الدرجة الاولى هي (1) طالما ان الفرق الاول لكل منها متكمل من الدرجة صفر. 3. اختبار النكامل المشترك بعد التأكد من ان بيانات السلاسل الزمية للمتغيرات موضع البحث بأنها مستقرة في الفرق الاول بأستخدام اختبار (ديكي فولرADF ) فأن التوصيف لهذه العلاقة طويالة الامد يتطلب اختبار ثاني وهو اختبار التكمل المشترك للمتغيرات الاساسية الداخلة في البحث. بعد النظر المى نتائُ اختبار جذور الوحدة، سوف نقوم بتقدير للتكامل المشترك باستخدام اختبار (جوهانسن).

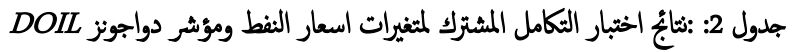

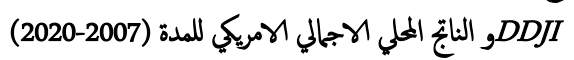

\begin{tabular}{|c|c|c|c|c|}
\hline \multicolumn{5}{|c|}{ Test (Trace) $\boldsymbol{\lambda}$} \\
\hline Eigenvalue & $\begin{array}{c}0.05 \\
\text { Critical } \\
\text { Value }\end{array}$ & $\begin{array}{l}\boldsymbol{\lambda}_{\text {trace }} \\
\text { Statistic }\end{array}$ & Prob. $^{* *}$ & $\begin{array}{c}\text { Null } \\
\text { hypothesis }\end{array}$ \\
\hline 0.139839 & 42.91525 & 51.48504 & 0.0056 & $r=0$ \\
\hline 0.122550 & 25.87211 & 27.53403 & 0.0308 & $\mathrm{r}=0$ \\
\hline 0.041547 & 12.51798 & 6.747155 & 0.3716 & $\mathrm{r} \leq 1$ \\
\hline \multicolumn{5}{|c|}{ Maximum Eigenvalue $\boldsymbol{\lambda}$ Max } \\
\hline Eigenvalue & $\begin{array}{c}0.05 \\
\text { Critical } \\
\text { Value }\end{array}$ & $\begin{array}{l}\boldsymbol{\lambda}_{\text {trace }} \\
\text { Statistic }\end{array}$ & Prob. $^{* *}$ & $\begin{array}{c}\text { Null } \\
\text { hypothesis }\end{array}$ \\
\hline 0.139839 & 22.82321 & 25.95101 & 0.0466 & $\mathrm{r}=0$ \\
\hline 0.122550 & 19.38704 & 20.78687 & 0.0312 & $\mathrm{r}=0$ \\
\hline 0.041547 & 12.51798 & 6.747155 & 0.3716 & $\mathrm{r} \leq 1$ \\
\hline
\end{tabular}

بالنظر الى جدول رقة (2) لنتائُ اختبار جوهانسن للنكامل المشترك باستخدام دالة الهمكن الاعظم لمتغيرات اسعار النفط ومؤشر دواجونز والناتج المحلي الاجالي الامريكي ، بينت النتاجُ ان القيمة المحسوبة لكل من اختبار احصاءة الاثر بلغت (trace=51.48504) وهي اكبر من القيمة الحرجة عند مستوى لك max معنوية 5\% وكما موضح في الجدول (2) اما فيا يخص اختبار قيمة العظمى eigenvalue فقد بلغت احصاء القيمة العظمى (25.95101) وهي اكبر من القيمة الحرجة عند مستوى معنوي 5\%، اي اننا في الاختباريين نرفض فرضية العدم تفتزض ان r=0، ونتبل بفرضية البديلة التي تنص على وجود تكامل 


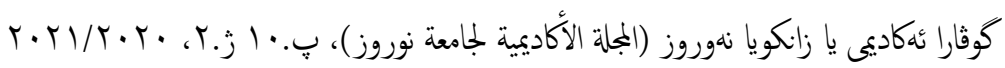

معنوية معلمة USA_GDP في التأثير على مؤشر داووجونز حيث بلغت

قيمة t المحتسبة (13.00) وهي مستوى 1\%.

F- بالنسبة لاختبار معادلة الانحدار الخطي المتعدد لاحظ ان ققمة statistic المحسوبة جاءت مساوية ل (135.0197) وهي قيمة جوهرية عند اي مستوى معنوية، واثبت ذلك قيمة P-value التي جاءت مساوية ل (0.000) وكما هو مبين في الجدول اعلاه، وهو الامر الذي من خلاله نستطع رفض فرض العدم لصاح الفرض البديل القائل بان واحد على الاقل من معاملات الانحدار يختلف معنوياً عن الصفر والبتالي جوهرية

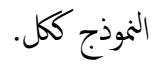

اما بالنسبة لمدى مساهمة ثاثير المتغير المستقل في تحديد سلوك المتغير التابع، تبين لنا من قيمة معامل التحديد R-squared التي جاءت مساوية ل (0.62) اي ان 62\% من التغيرات الحاصل في المتغير التابع هو (اسعار

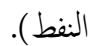
قيمة الاحصائية Durbin-Watson stat والتي بلغت (2.01431) وهي قيمة فوق (2)، مما يدل على عدم وجود مشكلة الارتباط الذاتي بين البواتي وعليه ستقبل فرضية العدم وترفض فرضية البديلة لكون فرضية العدم تنص على عدم وجود مشكلة الارتباط الناتي.

جدول 6: تقدير اثر اسعار النطط والناتج الملي الاجالي الياباني على مؤشر نيكي الياباني (2020-2007) (للمدIKKEI225

Dependent Variable: NIKKEI225

Method: Least Squares

Sample: 1164

\begin{tabular}{ccccc}
\hline Variable & Coefficient & Std. Error & t-Statistic & Prob. \\
\hline OIL & -58.62314 & 13.96078 & -4.199130 & 0.0000 \\
\hline JAPAN_GDP & $-5.07 \mathrm{E}-09$ & $6.79 \mathrm{E}-10$ & -7.459678 & 0.0000 \\
\hline C & 45831.82 & 3108.556 & 14.74377 & 0.0000 \\
\hline R-squared & 0.515350 & Mean dependent var & 15465.0 \\
\hline Adjusted R-squared & 0.488833 & S.D. dependent var & 4913.62 \\
\hline S.E. of regression & 3581.108 & Akaike info criterion & 19.2228 \\
\hline Sum squared resid & $2.06 \mathrm{E}+09$ & Schwarz criterion & 19.2795 \\
\hline Log likelihood & -1573.274 & Hannan-Quinn criter. & 19.2458 \\
\hline F-statistic & 72.93566 & Durbin-Watson stat & .91951 \\
\hline Prob(F-statistic) & 0.000000 & & \\
\hline \hline & Eviews8 & & \\
\hline
\end{tabular}

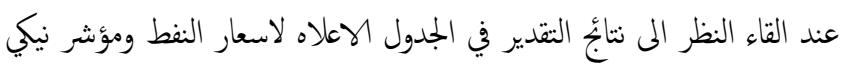

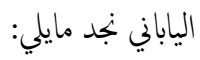

اما في ميتعلق لمتغري اسعار النفط ومؤشر ستوكي الاوربي والناتج المحلي الاجالي منطقة يورو وبالنظر الى جدول رقز (4) لنتائج اختبار جوهانسن للتكامل المشترك باستخدام دالة الومكان الوعظم لمتغيري مذكور انفا، بينت النتائج ان القيمة المسوبة لكل من اختبار احصاءة الاثر بلغت \%5 وهي اكبر من القيمة الحرجة عند مستوى معنوية (trace=30.48350) وكما موضح في الجدول(4) أما فيما يخص اختبار قيمة العظى ستى فقد بلغت احصاء القيمة العظىى (21.81760) وهي اكبر من القيمة الحرجة عند مستوى معنوي 5\%، اي انا في الاختباريين نرفض فرضية العدم تفترض ان rr=0 ونتبل بفرضية البديلة التي تنص على وجود تكامل مشترك.

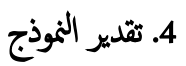
بعد استكمال كل من المرحلتيين وهما استقرارية السلاسل الزمنية للمتغيرات وتحويل السلاسل الزمنية غير المستقرة الى مستقرة بعد التحقق منها، ومعرفة درجة النكامل المشترك بين المتغيرات، وبالاعتاد استخدام لطريقة الانموذج الخطي في الخصول على معلمات دقيقة، نذهب الى الخطوة التالية وهي تقدير، وكانت التنائُ بالنسبة للمتغير المستقل وهو النفط والمتغير التابع مؤشر داوجونز الامريكة والناتج المحلي الاجالي الامريكي جاءت نحو الاتي: جدول 5: تقدير اثر اسعار النفط و الناجج الملي الاجلي الامريكي على مؤشر داووجونز (2020-2007) (لمد DDI

Dependent Variable: DJI

Method: Least Squares

Sample: 1164

\begin{tabular}{ccccc}
\hline Variable & Coefficient & Std. Error & t-Statistic & Prob. \\
\hline OIL & -23.12608 & 13.69583 & $.6885492-$ & 3240.0 \\
\hline USA_GDP & $1.65 E-09$ & $1.27 \mathrm{E}-10$ & 13.00913 & 0.0000 \\
\hline C & -13617.18 & 3104.039 & -4.386922 & 0.0000 \\
\hline R-squared & 0.626484 & Mean 16618.84 \\
\hline Adjusted R-squared & 0.621844 & S.D. dependent var & 5697.546 \\
\hline S.E. of regression & 3503.670 & Akaike info criterion & 19.1791 \\
\hline Sum squared resid & $1.98 \mathrm{E}+09$ & Schwarz criterion & 19.2358 \\
\hline Log likelihood & -1569.689 & Hannan-Quinn criter. & 19.2021 \\
\hline F-statistic & 135.0197 & Durbin-Watson stat & 2.01431 \\
\hline Prob(F-statistic) & 0.000000 & & \\
\hline \hline
\end{tabular}

عند القاء النظر الى نتائج التقدير في الجدول الهعلاه لاسعار النفط ومؤشر

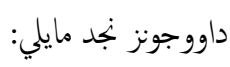

اثبت اختبار t معنوية معلمة النفط في الثأثير على مؤشر داووجونز حيث بلغت قيمة t t2.688) وهي معنوية عند مستوى 5\% 


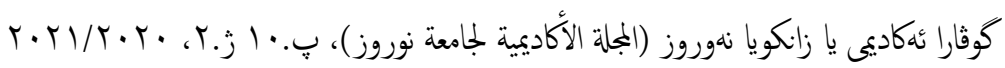

عند القاء النظر الى نتاجُ التقدير في الجدول الاعلاه لاسعار النفط ومؤشر

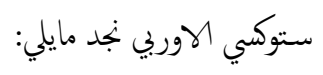

اثبت اختبار t عدم معنوية معلمة النفط في التأثير على مؤشر ستوكيي حيث بلغت قيمة t (1.5) وهي غير معنوية عند مستويات المقبولة. وكذلك ثبتة عدم معنوية معلمة EUROPEAN_UNION _GDP في الثأثير على مؤشر نيكي حيث بلغت قيمة t المتسبة (1.17) وهي غير معنوية عند

$$
\text { مستويات المقبولة. }
$$

F- بالنسبة لاختبار معادلة الانحدار الخطي البسيط نلاحظ ان قيمة statistic مستويات 1\%، 5\%، واثبت ذلك قيمة P-value التي جاءت مساوية ل(0.059) وكما هو مبين في الجدول اعلاه، وهو الامر الذي من خلاله نستطيع قبول فرضية العدم لصالح ورفض فرضية البديلة وبالتالي عدم

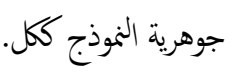
اما بالنسبة لمدى مساهمة ناثير المتغير المستقل في تحديد سلوك المتغير التابع، تبين لنا من قيمة معامل التحديد R-squared التي جاءت مساوية ل (0.0287) اي ان 2\% من التغيرات الحاصل في المتغير التابع هو

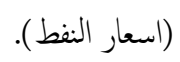

قيمة الاحصائية Durbin-Watson stat والتي بلغت (0.095928) وهي قيمة بعيدة عن (2)، مما يدل على وجود مشكلة الارتباط الذاتي بين البواقي وعليه نرفض فرضية العدم ونتبل فرضية البديلة لكون فرضية العدم

$$
\text { تنص على عدم وجود مشكلة الارتباط الذاتي. }
$$

جدول 8: العلاقة السبية بين اسعار النفط ومؤشر داووجونز DDJI و الناجّ المحلي الاجلالي الامريكي للمدة (2007-2020)

Pairwise Granger Causality Tests

Date: 04/26/21 Time: 22:51

Sample: 1168

\begin{tabular}{lrrr} 
Lags: 3 & & & \\
\hline Null Hypothesis: & Obs & F-Statistic & Prob. \\
\hline OIL does not Granger Cause DJI & 161 & 2.73613 & 0.0455 \\
\hline DJI does not Granger Cause OIL & & 3.07967 & 0.0293 \\
\hline USA_GDP does not Granger Cause DJI & 161 & 0.37088 & 0.7741 \\
\hline DJI does not Granger Cause USA_GDP & & 2.16636 & 0.0943 \\
\hline USA_GDP does not Granger Cause OIL & 161 & 0.47539 & 0.6999 \\
\hline OIL does not Granger Cause USA_GDP & & 0.44133 & 0.7238 \\
\hline
\end{tabular}

اثبت اختبار t معنوية معلمة النفط في الثأثير على مؤشر نيكي حيث بلغت قيمة t t4.2) وهي معنوية عند مستوى 1\%. وكذلك ثبتة معنوية معلمة t في التأثير على مؤشر نيكي حيث بلغت قيمة JAPAN _GDP المتسبة (7.50) وهي مستوى 1\%.

F- بالنسبة لاختبار معادلة الانحدار الخطي البسيط نلاحظ ان قيمة statistic عند اي مستوى معنوية، واثبت ذلك قيمة P-value التي جاءت مساوية للصفر وكما هو مبين في الجدول اعلاه، وهو الامر الذي من خلاله نستطيع رفض فرض العدم لصاح الفرض البديل القائل بان واحد على الاقل من معاملات الانحدار يختلف معنوياً عن الصفر والبنالي جوهرية تأثير المتغير المستقل على المتغير التابع. اما بالنسبة لمدى مساهمة تاثير المتغير المستقل في تحديد سلوك المتغير التابع، تبين لنا من قيمة معامل التحديد R-squared التي جاءت مساوية ل (0.515350) اي ان 51\% من التغيرات الحاصل في المتغبر التابع هو (اسعار النفط). (- - (اسط) قيمة الاحصائية Durbin-Watson stat والتي بلغت (1.9195) وهي قيمة قريبة من (2)، مما يدل على عدم وجود مشكلة الارتباط الذاتي بين البواقي وعليه ستقبل فرضية العدم وترفض فرضية البديلة لكون فرضية العدم تنص على عدم وجود مشكلة الارتباط الذاتي. جدول (7): تقدير اثر اسعار الثفط والناتج المللي الاجلالي الياباني على مؤشر ستوكيي

\begin{tabular}{|c|c|c|}
\hline \multicolumn{3}{|c|}{$\begin{array}{c}\text { Dependent Variable: STOXX50E } \\
\text { Method: Least Squares } \\
\text { Included observations: } 164\end{array}$} \\
\hline Variable & Coefficient Std. Error t-Statistic & Prob. \\
\hline OIL & $\begin{array}{llll}-2.746833 & 1.835816 & -1.496246\end{array}$ & 0.1365 \\
\hline EUROPEAN_UNION_GDP & $-8.11 \mathrm{E}-11 \quad 6.95 \mathrm{E}-11 \quad-1.167122$ & 0.2449 \\
\hline $\mathrm{C}$ & $\begin{array}{llll}4552.389 & 1020.054 & 4.462891\end{array}$ & 0.0000 \\
\hline R-squared & 0.028703 Mean dependent var & 3135.126 \\
\hline Adjusted R-squared & 0.016637 S.D. dependent var & 539.6604 \\
\hline S.E. of regression & 535.1524 Akaike info criterion & 15.42110 \\
\hline \multirow[t]{2}{*}{ Sum squared resid } & 46108490 Schwarz criterion & 15.47781 \\
\hline & Hannan-Quinn & \\
\hline Log likelihood & -1261.531 & 15.44412 \\
\hline F-statistic & 2.378833 Durbin-Watson stat & 0.095928 \\
\hline Prob(F-statistic) & 0.095908 & \\
\hline
\end{tabular}
الاوريي STOXX50E والناتج الملي الاججالي للاتحاد الاوريي للمدة (72007-2020) 
من خلال اختبار السبية الموضح في الجدول (10) نلاحظ وجود اتجاه واحد للسبية من اسعار النفط الى ومؤشر ستوكسي اي ان سعر النفط يؤثر على مؤشر ستوكي، ولم تثبت اي علاقة سبية اخرى بين بقية المتغرات. 5. 5 5 الاستناجات و المقترحات 1.5 توصل البحث الى الاستنتاجات مايلي ان النفط يجري تداوله والمضاربة بيه في الاسواق المالية العالمية والمضاربات في اسعار النفط تخضع في كثير من الاحيان للعقود الاجله وان هذه المضاربات تستند الى التوقعات وهذه تشمل كل الفرص الاستثارية

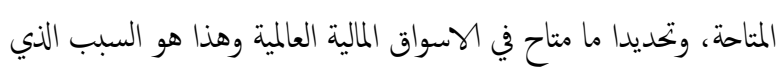
يجعل يقوي علاقة الارتباط بين الاسواق المالية واسواق النفطية. اثبت الاختبار القياسي ان اسعار النفط توثثر ايجابا على مؤشرات الاسواق المالية قيد الدراسة (مؤشر داوو جونز الامريكي DJI ومؤشرنيكلي الياباني NIKKEI225) اي ان زيادة اسعار النفط تؤدي الى ارتفاع مؤشرات الاسواق المالية. ولم يثبت تأثير اسعار النفط على ومؤشر ستوكي الاتحاد الاوربي .STOXX50E اثبتت الاختبارات السبية ان هنالك اتجاهين لسبية بين مؤشر داووجونز الاميكي والنفط في حين كان اتجاه السبية من اسعار النفط الى مؤشري (ومؤشرنيكاي الياباني NIKKEI225، مؤشر ستوكي الاتحاد الاوربي STOXX50E). ان عدم تأثير اسعار النفط على المؤشر الووريي يككن ان يعزا المى اسباب متعددة منها التوسع في استخدامات الطاقات البديلة فضلا عن ان المضاربات بالنفط في الاسواق المالية الاوربية شهدة تراجعاً امام الانواع الاخرى من المضاربات في الاسنوات الاخيرة وخاصتاً منها بالعقود الاجلة وهو يعبر عن اختلاف في سلوك المضارب الاوربي عن ما موجود لدى

$$
\text { المضارب الامريكي والياباني. }
$$

ضرورة العمل المستمر على قياس تاثيرات اسعار النفط على اسواق المالية واستخدام الاساليب القياسية والاحصائية الحديثة في هذا المجال لان
من خلال اختبار السبية الموضح في الجدول (8) نلاحظ وجود اتجاهين للسببية بين اسعار ومؤشر داووجونز اي ان سعر النفط يؤثر وتأثر بمؤشر داووجونز ، اما

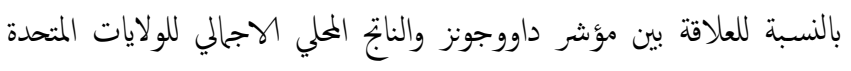
الامريكة هنالك ضعف في العلاقة السبية والامر ينطبق على العلاقة بين النفط والناتج المحلي الاجالي. جدول 9: العلاقة السبية بين اسعار النفط ومؤشر نيكي الياباني NIKKEI225 و النابج الملي الاججالي الياباني للمدة (2007-2020)

Pairwise Granger Causality Tests

Date: 04/26/21 Time: 22:54

Sample: 1168

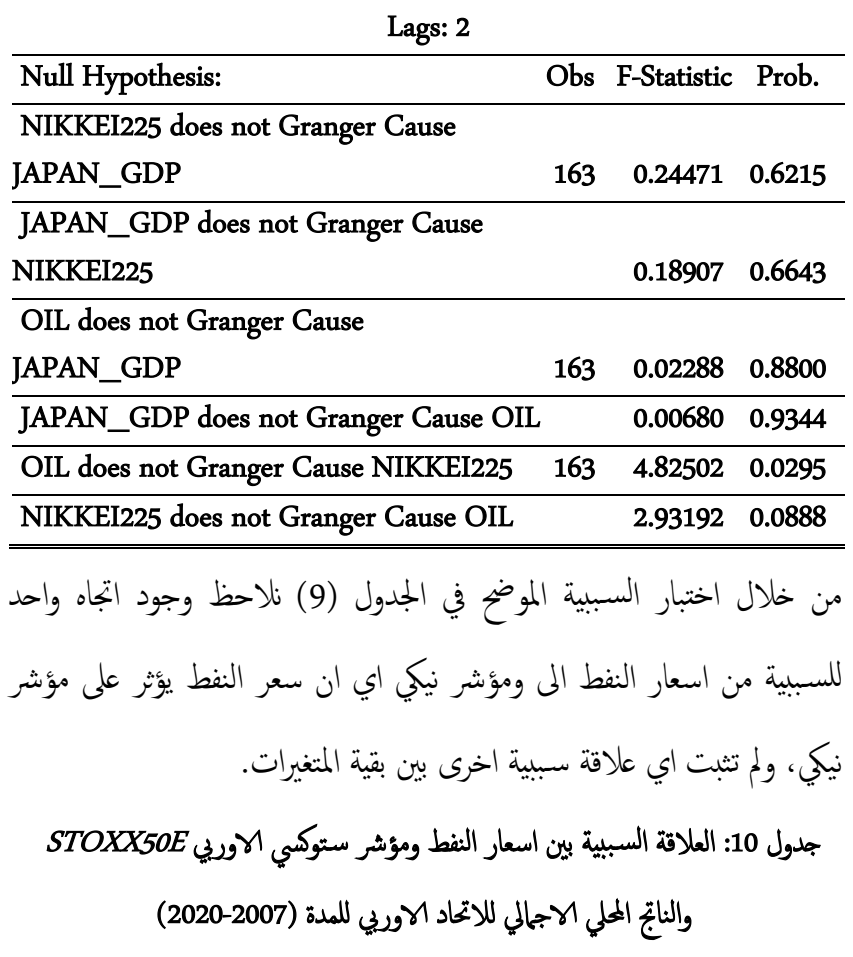

Pairwise Granger Causality Tests

Date: 04/26/21 Time: 22:57

Sample: 1168

Lags: 3

Null Hypothesis: Obs F-Statistic Prob.

EURO_AREA_GDP does not

$\begin{array}{llll}\text { Granger Cause OIL } & 161 & 0.23085 & 0.8748\end{array}$

OIL does not Granger Cause

EURO_AREA_GDP

$0.35815 \quad 0.7833$

STOXX50E does not Granger

Cause OIL

$\begin{array}{lll}161 & 1.14577 & 0.3326\end{array}$

OIL does not Granger Cause

STOXX50E

$3.46531 \quad 0.0178$

STOXX50E does not Granger

Cause EURO_AREA_GDP $\quad 161 \quad 1.77289 \quad 0.1547$

EURO_AREA_GDP does not

Granger Cause STOXX50E $\quad 0.45196 \quad 0.7163$ 


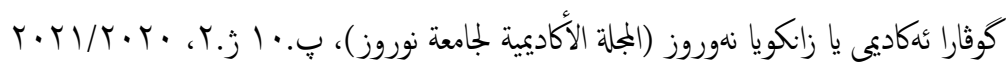

indicators: Copula approach, ELSEVIER, Energy Economics Volume 61, January 2017, Pages 162-173.

3. Stavros Degiannakis, George Filis, and Renatas Kizys, The Effects of Oil Price Shocks on Stock Market Volatility: Evidence from European Data, The Energy Journal, 2014, vol. Volume 35, issue Number 1.

4. Enitan Odupitan, EFFECTS OF CRASHING CRUDE OIL PRICES ON OIL PRODUCING COUNTRIES, CENTRIA UNIVERSITY OF APPLIED SCIENCES, Degree Programme in Business Management, December 2017

5. Reinhard Ellwanger, Benjamin Sawatzky and Konrad Zmitrowicz, Factors Behind the 2014 Oil Price Decline, International Economic Analysis Department, Bank of Canada Review, 2017

6. Ehenbo Hou, Jodiekeane, Jane Kennan and Dirk willem, the oil price shock of impacts and policy implication, shaping policy for development (Odiorg) march 2015.

7. ECB Economic Bulletin, Are the recent oil price increases set to last?, Issue 2 / 2018 - Boxes.

8. PETROLEUM ECONOMIST, Independent Analysis for Energy Leaders .The rise and fall of oil prices in 2018. Outlook 2019

$$
\begin{aligned}
& \text { متغير اسعار النفط يعد من المتغيرات المهمة على المستوى العالمي وان } \\
& \text { تأثيراته تختلف حسب وضع الطاقة في اي دولة. }
\end{aligned}
$$

التشابكات القطاعية والترابطات والثأثيرات المتبادلة امور يصعب قياسها

بأستخدام اسلوب قياسي واحد لذلك القياس الاقتصادي الدقيق لهذه

ترابطات قد يجتاج الى تطوير نماذج خاصة بالدول وبقطاع الطاقة تحديداً

$$
\text { وترابطاته الامامية والخلفية. }
$$

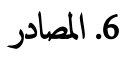

1.6 البحوث العربية

1. بالنور هاجر، منصر سامية، فاطمة بوجريدة، أثر تقلبات أسعار النفط على عوائد

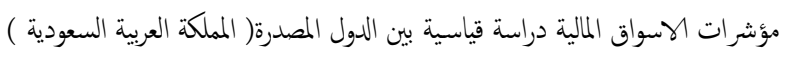

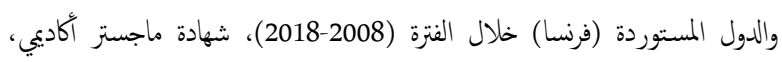

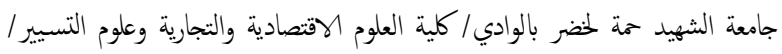

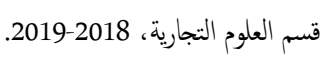

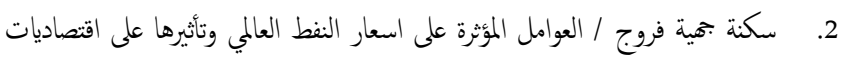

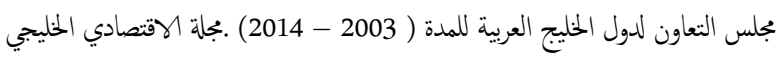
/ العدد 22، حزيران 2015. 3. الدكتور أحمد إبرهي علي, تحليل سوق النفط العالمي, البنك المركزي العراقي، اذار 2016 4. علي ميرزا، ندوة (تداعيات هبوط اسعار النفط على البلدان المصدرة)/ مركز العربي

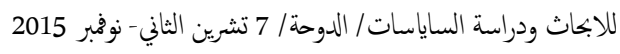

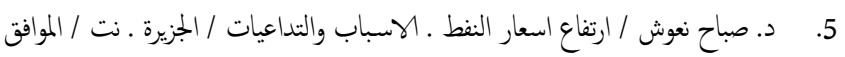
2008/6/30 الساعة 11: 39 . 39 6. د. محمد الشطي, ما هي فرص تكرار سيناريو أسعار النفط في عام 2008؟, مجلة .

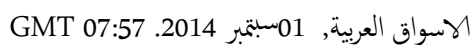
7.

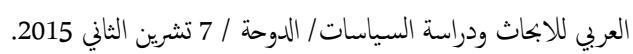
8.

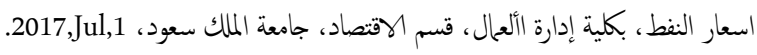

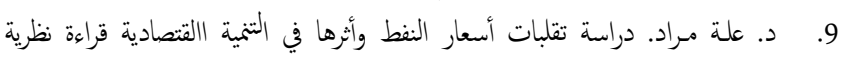
تحليلية في حالة الجزائر للفترة عام 2000-2014، جامعة زيان عاشور بالجلفة. 2017 يناير. 10. ( منظمة الاقطار العربية المصدرة للبترول ( اوبك) / دراسة حول تطور خارطة سوق

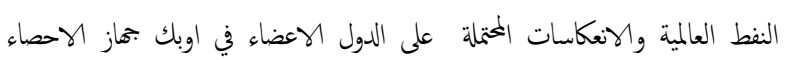

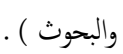

\section{6 البحوث الهكلينية}

1. (TaheriNoushin, the Impact of Oil Price on Stock Markets: Evidence from Developed Markets, Eastern Mediterranean University January 2014).

2. Derya Ezgi Kayalar a, C. Coşkun Küçüközmen b, A. Sevtap Selcuk-Kestel, The impact of crude oil prices on financial market 